\title{
Experimental stabilization of transverse collective instabilities in the LHC with second order chromaticity
}

\author{
M. Schenk, ${ }^{1,2, *}$ X. Buffat, ${ }^{1}$ L. R. Carver, ${ }^{1}$ R. De Maria, ${ }^{1}$ K. Li, ${ }^{1}$ and E. Métral ${ }^{1}$ \\ ${ }^{1}$ European Organization for Nuclear Research (CERN), CH-1211 Geneva, Switzerland \\ ${ }^{2}$ École Polytechnique Fédérale de Lausanne (EPFL), CH-1015 Lausanne, Switzerland
}

(Received 8 March 2018; published 17 August 2018)

\begin{abstract}
This paper discusses measurements on the stabilization of single bunches with second order chromaticity $\left(Q^{\prime \prime}\right)$ in the Large Hadron Collider (LHC) at CERN. $Q^{\prime \prime}$ introduces an incoherent betatron tune spread which can produce Landau damping of transverse instabilities. Although the resulting stabilizing effect is similar to that provided by Landau octupoles, the underlying beam dynamics are different. Since the tune spread from $Q^{\prime \prime}$ is based on the longitudinal rather than the transverse action of the particles, it will not be affected by the smaller transverse emittance beams of future machines, such as the High Luminosity LHC or the Future Circular Collider, and may hence provide more efficient Landau damping than magnetic octupoles. This study serves as a proof-of-principle experiment to demonstrate Landau damping from detuning with longitudinal action by means of $Q^{\prime \prime}$ in a carefully prepared and well-understood accelerator environment. The agreement between measurements and PyHEADTAIL tracking simulations shows that $Q^{\prime \prime}$ indeed contributes to the beam stability, that the numerical model of the LHC is accurate, and that the involved beam dynamics mechanisms are understood from both the single- and multiparticle effects points of view. The results also serve as a first experimental validation of the recently proposed radio frequency quadrupole for Landau damping.
\end{abstract}

DOI: 10.1103/PhysRevAccelBeams.21.084401

\section{INTRODUCTION}

Landau damping is a powerful stabilizing mechanism and is commonly employed to mitigate transverse collective instabilities in particle colliders [1,2]. The mechanism is present when there is an incoherent spread in the betatron tunes $Q_{x, y}$ of the particles in the beam. The spread is a result of machine nonlinearities often introduced by design through dedicated elements. In the Large Hadron Collider (LHC), magnetic octupoles are installed to produce an incoherent tune spread that depends on the transverse actions of the particles [3-5]. These Landau octupoles are extensively used for beam stabilization during LHC operation, in particular, to suppress impedance-driven head-tail instabilities before the beams are brought into collision [6,7].

Recently, the design and working principle of a radio frequency (rf) quadrupole has been published, including a numerical analysis of its stabilizing effect [8-10]. Unlike Landau octupoles, an rf quadrupole generates a betatron tune spread depending on the longitudinal rather than the

*michael.schenk@cern.ch

Published by the American Physical Society under the terms of the Creative Commons Attribution 4.0 International license. Further distribution of this work must maintain attribution to the author(s) and the published article's title, journal citation, and DOI. transverse actions. This approach provides potentially a more effective means for stabilization of the beams of future, high-energy hadron colliders. The reason is that Landau octupoles will be less effective for smaller transverse emittance beams because of the reduced transverse action spreads [10]. The longitudinal action spread, on the other hand, remains of the same order of magnitude, and hence the betatron detuning with an rf quadrupole can be very effective $[8,10]$. For example, the high luminosity upgrade of the LHC will operate with beams with a ratio of $10^{-4}$ between the one-sigma transverse and longitudinal emittances at $7 \mathrm{TeV}$ [11]. For the Future Circular Collider, this number becomes another 10-100 times smaller at the top energy (50 TeV), reducing further the effect of Landau octupoles [12]. It is thus advisable to explore alternative methods that could provide Landau damping in future machines.

The second order chromaticity $Q_{x, y}^{\prime \prime}=\partial^{2} Q_{x, y} /\left.\partial \delta^{2}\right|_{\delta=0}$, where $\delta=d p / p$ is the relative momentum error, generates an incoherent betatron tune spread as a function of longitudinal action in the same manner as the rf quadrupole as will be derived in this paper. Indeed, it can be shown that the detuning introduced by $Q^{\prime \prime}$ mimics the effect of the $\mathrm{rf}$ quadrupole in a first approximation. The advantage of second order chromaticity is that it can be introduced in an existing machine like the LHC without the need for installing new hardware, for example, by powering the different families of the main sextupoles in a specific 
configuration. Despite the optics constraints which can limit the achievable dynamic range of $Q^{\prime \prime}$, second order chromaticity still provides a practical and cost-effective way for a first experimental verification of the stabilizing effect expected from an rf quadrupole cavity.

The main objective of this paper is to present a proof-ofprinciple experiment that confirms the stabilizing effect of transverse detuning with longitudinal amplitude and to validate the beam dynamics models. The observations made in LHC experiments on single-bunch stability with $Q^{\prime \prime}$ will be used to demonstrate that second order chromaticity has a stabilizing effect and that the main beam dynamics effects are understood and correctly modeled in numerical simulation codes such as MAD-X and PyHEADTAIL, from both the single- and the multiparticle dynamics points of view $[13,14]$. Section II briefly summarizes the detuning mechanisms for $Q^{\prime \prime}$ and the rf quadrupole and shows the equivalence of the two approaches in a first approximation. Section III describes the production scheme for $Q^{\prime \prime}$ and the definition of two dedicated knobs to power the LHC main sextupole magnets in a specific configuration. This includes numerical calculations and an analysis of undesired side effects. Section IV discusses the characteristics of the singlebunch head-tail mode in the LHC at the top energy (6.5 TeV) and how this instability is routinely mitigated by means of the Landau octupoles in the absence of the $Q^{\prime \prime}$ knobs. The analysis is done using both experimental data and results from the macroparticle tracking code PyHEADTAIL. It demonstrates that the simulations successfully reproduce the beam dynamics in the machine. Finally, Sec. V details the observations made during dedicated measurements with the $Q^{\prime \prime}$ knobs defined in Sec. III. Again by means of PyHEADTAIL simulations, the involved beam dynamics mechanisms are clearly identified and understood. Among others, the differences between stabilizing the single bunch with Landau octupoles or with $Q^{\prime \prime}$ become evident.

\section{ANALYTICAL CONSIDERATIONS}

The purpose of this section is twofold: first, to show that the incoherent betatron detuning produced by $Q^{\prime \prime}$ depends on the longitudinal action of the particles and, second, to briefly review the tune spread introduced by an rf quadrupole and to prove that it is equivalent to $Q^{\prime \prime}$ in a first approximation.

\section{A. Incoherent tune spread from $Q^{\prime \prime}$}

Given a machine lattice with first and second order chromaticities $Q_{x, y}^{\prime}$ and $Q_{x, y}^{\prime \prime}$, a particle $i$ with a relative momentum deviation of $\delta_{i}$ experiences a betatron tune shift:

$$
\Delta Q_{x, y}^{i}\left(\delta_{i}\right)=Q_{x, y}^{\prime} \delta_{i}+\frac{Q_{x, y}^{\prime \prime}}{2} \delta_{i}^{2} .
$$

Since the particle undergoes synchrotron motion, its momentum deviation varies over time and takes on values in the interval $\left[-\hat{\delta}_{i}, \hat{\delta}_{i}\right]$, where $\hat{\delta}_{i}$ is the maximum momentum deviation amplitude of the synchrotron oscillation. By consequence, $\Delta Q_{x, y}^{i}$ has an implicit time dependence, and the effective incoherent detuning of the particle is given by the time average $\left\langle\Delta Q_{x, y}^{i}\left[\delta_{i}(t)\right]\right\rangle_{t \rightarrow \infty}$, in analogy to detuning with the transverse amplitude from magnetic octupoles [5]. Assuming linear synchrotron motion

$$
\delta_{i}(t)=\hat{\delta}_{i} \cos \left(Q_{s} \omega_{0} t+\phi_{\delta, i}\right),
$$

where $Q_{s}$ is the synchrotron tune, $\omega_{0}$ the angular revolution frequency, and $\phi_{\delta, i}$ the constant synchrotron phase offset of the particle, the calculation of the effective detuning becomes straightforward. It is obtained by averaging Eq. (1) over one synchrotron period $T_{s}=2 \pi / Q_{s} \omega_{0}$ :

$$
\begin{aligned}
\left\langle\Delta Q_{x, y}^{i}\right\rangle & =\frac{Q_{x, y}^{\prime \prime} \hat{\delta}_{i}^{2}}{2} \frac{1}{T_{s}} \int_{0}^{T_{s}} \cos ^{2}\left(Q_{s} \omega_{0} t+\phi_{\delta, i}\right) d t \\
& =\frac{Q_{x, y}^{\prime \prime} \hat{\delta}_{i}^{2}}{4} .
\end{aligned}
$$

This is equivalent to averaging for $t \rightarrow \infty$ as described in Ref. [5], given that $Q_{s}$ is not a rational number. $\hat{\delta}_{i}^{2}$ can be rewritten in terms of the longitudinal action $J_{z}^{i}$ of the particle, $\hat{\delta}_{i}^{2}=2 J_{z}^{i} Q_{s} / \eta R$, with $\eta$ the slip factor and $R$ the physical radius of the accelerator ring. This leads to the final expression

$$
\left\langle\Delta Q_{x, y}^{i}\right\rangle=\frac{Q_{x, y}^{\prime \prime}}{2} \frac{Q_{s}}{\eta R} J_{z}^{i} \doteq \alpha_{z}^{x, y} J_{z}^{i}
$$

where

$$
\alpha_{z}^{x, y}=\partial\left\langle\Delta Q_{x, y}^{i}\right\rangle / \partial J_{z}^{i}=Q_{x, y}^{\prime \prime} Q_{s} / 2 \eta R
$$

is the longitudinal detuning coefficient, defined in analogy to detuning with the transverse amplitude.

An approximate dispersion relation specifically for detuning with longitudinal amplitude has been derived by Berg and Ruggiero [15]. Their work shows that the incoherent tune spread leads to an increase of the stable region in the complex tune space $\operatorname{Re}\left(\Delta Q_{\text {coh }}\right)$ vs $-\operatorname{Im}\left(\Delta Q_{\text {coh }}\right)$ in equivalence to Landau damping from magnetic octupoles. Here $\Delta Q_{\text {coh }}$ denotes the complex coherent tune shift that characterizes a particular head-tail instability. The real part is given by the tune shift of the bunch centroid oscillation with respect to the unperturbed tune of the unstable synchrotron sideband. The imaginary part corresponds to the exponential growth rate of the amplitude of the bunch centroid motion. Two incoherent tune distributions and their corresponding stability diagrams are shown in the upper and lower plot in Fig. 1, respectively, one for positive (blue) and one for negative (red) $Q^{\prime \prime}$, respectively. An azimuthal mode zero head-tail instability was assumed for computing the stability 

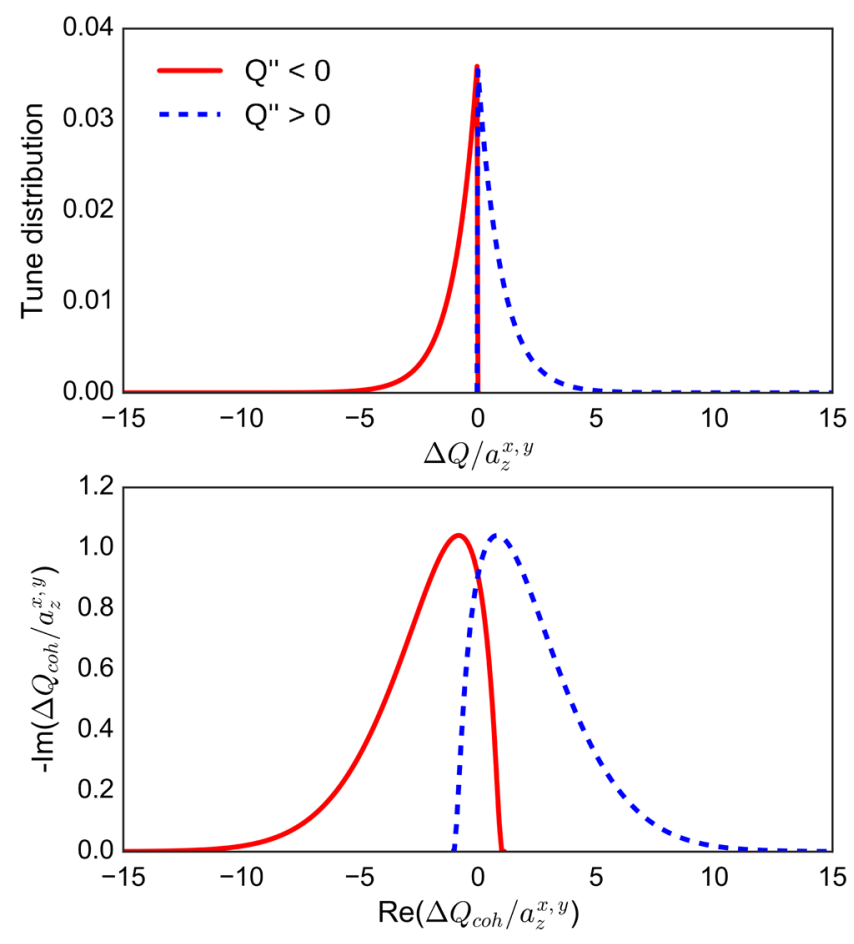

FIG. 1. Incoherent tune distributions (top) and normalized stability diagrams (bottom) for detuning with the longitudinal amplitude from $Q^{\prime \prime}<0$ (red, solid line) or $Q^{\prime \prime}>0$ (blue, dashed line), respectively, computed employing the formalism described in Ref. [15].

diagrams. The way to read the plots is that all the head-tail instabilities with an unperturbed coherent tune shift, i.e., measured in the absence of any tune spread, situated below the line traced out by the stability diagram will be Landau damped. The asymmetry of the stable regions for the two signs of $Q^{\prime \prime}$ is a result of the strictly one-sided detuning [see Eq. (3)].

It is worth noting that the average detuning in Eq. (2) contains only the term with $Q_{x, y}^{\prime \prime}$. The first order chromaticity does not contribute to the effective tune spread and, hence, does not introduce Landau damping. It does, however, change the effective impedance, i.e., the overlap sum between the beam spectrum and the impedance, and can hence modify the head-tail instability mechanism. As such, it can, for example, be used to raise the transverse mode coupling instability threshold [2]. A similar change of the effective impedance is also observed for $Q_{x, y}^{\prime \prime}$, in the theory, simulations, and experiments (see also Sec. V). This effect often plays an important role in addition to the Landau damping.

\section{B. Equivalence to an rf quadrupole}

A particle $i$ that is subject to the transverse kicks from an rf quadrupole experiences a change of the betatron tunes $[8,10]$ :

$$
\Delta Q_{x, y}^{i}= \pm \beta_{x, y} \frac{b^{(2)}}{4 \pi B_{0} \rho} \cos \left(\frac{\omega z_{i}}{\beta c}+\phi_{0}\right)
$$

$\beta_{x, y}$ are the transverse beta functions at the location of the device, $b^{(2)}$ is the rf quadrupolar integrated gradient in units of $(\mathrm{Tm} / \mathrm{m}), B_{0} \rho$ denotes the magnetic rigidity of the beam, $\omega$ is the angular frequency of the rf quadrupole field, $z_{i}$ is the longitudinal position of the particle measured with respect to the zero crossing of the main rf voltage, $\beta$ and $c$ denote the relativistic beta and the speed of light, respectively, and $\phi_{0}$ is a constant phase offset that determines the mode of operation of the rf quadrupole.

In Ref. [10], the authors show that if the wavelength of the rf wave is much larger than the bunch length $\sigma_{z}$, i.e., $\omega \sigma_{z} / \beta c \ll 1$, the effective tune spread provided by an $\mathrm{rf}$ quadrupole cavity (for $\phi_{0}=0$ ) reads

$$
\left\langle\Delta Q_{x, y}^{i}\right\rangle \approx \mp \beta_{x, y} \frac{b^{(2)}}{8 \pi B_{0} \rho}\left(\frac{\omega}{\beta c}\right)^{2} \frac{\eta R}{Q_{s}} J_{z}^{i} \doteq \tilde{\alpha}_{z}^{x, y} J_{z}^{i},
$$

where $\tilde{\alpha}_{z}^{x, y}$ has been defined as the longitudinal detuning coefficient for an if quadrupole

$$
\tilde{\alpha}_{z}^{x, y}=\mp \beta_{x, y} \frac{b^{(2)}}{8 \pi B_{0} \rho}\left(\frac{\omega}{\beta c}\right)^{2} \frac{\eta R}{Q_{s}},
$$

in analogy to $\alpha_{z}^{x, y}$ for detuning from $Q_{x, y}^{\prime \prime}$ in Eq. (3).

Equations (3) and (6) manifestly show the equivalence of $Q^{\prime \prime}$ and the rf quadrupole given the approximation $\omega \sigma_{z} / \beta c \ll 1$. Therefore, the betatron detuning introduced, respectively, by $Q^{\prime \prime}$ and an rf quadrupole is generated according to the same mechanism, and the effects on the beam dynamics are directly comparable. This makes $Q^{\prime \prime}$ a suitable tool for a first, cost-effective, experimental validation of the stabilizing effect from an rf quadrupole and also allows us to thoroughly benchmark the numerical models with beam measurements.

\section{III. $Q^{\prime \prime}$ IN THE LHC}

This section is split into three parts and contains information about how to generate and measure $Q^{\prime \prime}$ in the LHC. First, the sextupole powering scheme used to generate $Q^{\prime \prime}$ is analyzed by means of the LHC MAD-X model. Second, the detuning with the transverse amplitude introduced by the sextupoles is discussed and compared to the LHC Landau octupoles. The latter is a side effect that needs to be included in the study to decouple the different stabilizing mechanisms that are involved. Finally, the higher order chromaticity measurement method is explained and illustrated with example data.

\section{A. $Q^{\prime \prime}$ production scheme}

The second order chromaticity is an energy-dependent aberration that is defined primarily by the integral of the 
terms $\beta_{x, y}^{\prime}(\delta) k_{1}, \beta_{x, y}^{\prime}(\delta) D_{x} k_{2}$, and $D_{x}^{2} k_{3}$ around the machine lattice [16,17]. $k_{1}, k_{2}$, and $k_{3}$ are the quadrupolar, sextupolar, and octupolar strengths, respectively, $\beta_{x, y}^{\prime}=$ $\partial \beta_{x, y} / \partial \delta$ is the first derivative of the beta function with respect to the relative momentum deviation, and $D_{x}$ denotes the horizontal dispersion function. In the LHC, the main sextupoles (MS) are grouped into focusing (SF) and defocusing (SD) families, installed, respectively, at focusing and defocusing quadrupoles in the lattice. SF and SD are further divided into two interleaved subfamilies separated by a phase advance of approximately $\pi$. They are called $\left(F_{1}, F_{2}\right)$ and $\left(D_{1}, D_{2}\right)$, respectively. Each of the four families can be individually powered for each of the eight sectors of the LHC. With this sextupole scheme, $\beta_{x, y}^{\prime}(\delta)$ can be enhanced to correct, or introduce, $Q_{x, y}^{\prime \prime}$ without affecting the first order chromaticity $Q_{x, y}^{\prime}$. This is achieved by varying the strengths of the two subfamilies with opposite signs for both SF and SD separately in all the sectors $u \in$ $\{1,2, \ldots, 7,8\}$ of the machine:

$$
\begin{aligned}
\text { SF : } \Delta k_{2, F_{1}}^{u}=+b_{F}^{u} \Delta_{F}, & \Delta k_{2, F_{2}}^{u}=-b_{F}^{u} \Delta_{F}, \\
\text { SD: } \Delta k_{2, D_{1}}^{u}=+b_{D}^{u} \Delta_{D}, & \Delta k_{2, D_{2}}^{u}=-b_{D}^{u} \Delta_{D} .
\end{aligned}
$$

The absolute change of the sextupole strength $\Delta_{F(D)}$ is the same for all the SF (SD) in the lattice, but the families in each sector are powered with a different sign denoted by $b_{F(D)}^{u}$. With the definitions in Eq. (8), the absolute value of $Q_{x, y}^{\prime \prime}$ depends quadratically on the sextupolar strength, i.e., $\left|Q_{x, y}^{\prime \prime}\right| \propto \Delta_{F, D}^{2}[16,17]$. By choosing the signs $b_{F, D}^{u}$ carefully for each sector, one can define a set of almost orthogonal knobs to change $Q^{\prime \prime}$ towards positive or negative values in the two beams and for both planes independently. For the experiment in the LHC, the goal is to define a sextupole scheme that produces a large negative second order chromaticity in both transverse planes. The choice is based on the fact that the real part of the coherent tune shifts for the most unstable head-tail modes in the LHC is negative $[10,18]$. This is a result of the inductive nature of the collimator impedances which are the main contributors to the effective impedance at the top energy [18]. The stability diagrams displayed in the lower part in Fig. 1 demonstrate that in this case negative provides more effective Landau damping than positive $Q^{\prime \prime}$.

The best powering scheme to generate a significant amount of negative second order chromaticity for the LHC optics configuration used at the top energy has been determined by means of MAD-X/PTC $[3,14,19]$. The final results are listed in Table I. The two sextupole knobs are called QPPF and QPPD for the focusing and the defocusing sextupole families, respectively. The maximum possible variation in the sextupole strengths is given by $\Delta_{F, D}^{\max }$ together with the corresponding amounts of $Q_{x}^{\prime \prime}$ and $Q_{y}^{\prime \prime}$ expected from MAD-X calculations for both beam 1 and
TABLE I. Definition of the sextupole powering schemes used to introduce $Q_{x, y}^{\prime \prime}<0$ in the LHC at the top energy. For each of the two knobs QPPF and QPPD, the maximum strengths are listed together with the corresponding amounts of $Q^{\prime \prime}$ predicted by MAD- $X$ in the two beams $(B)$ and planes.

\begin{tabular}{cccccc}
\hline \hline Knob & $\mathrm{B}$ & $b_{F, D}^{(1,2, \ldots, 7,8)}$ & $\Delta_{F, D}^{\max }\left(\mathrm{m}^{-3}\right)$ & $10^{-4} Q_{x}^{\prime \prime}$ & $10^{-4} Q_{y}^{\prime \prime}$ \\
\hline QPPF & 1 & ++-+-+-- & 0.30 & -15.0 & -0.2 \\
& 2 & -++-++++ & 0.30 & -14.1 & 0.1 \\
QPPD & 1 & -+++-+++ & 0.28 & -0.1 & -8.9 \\
& 2 & ---++--+ & 0.28 & -0.1 & -4.3 \\
\hline \hline
\end{tabular}

beam 2. The values illustrate that the QPPF and QPPD knobs mainly produce $Q^{\prime \prime}$ in the horizontal and the vertical planes, respectively. The quadratic dependence of the second order chromaticity on the strength of the knobs is further illustrated by Fig. 2 for both beams and the two transverse planes. The plots also clearly demonstrate that the $Q^{\prime \prime}$ in the horizontal and the vertical planes can be controlled independently.
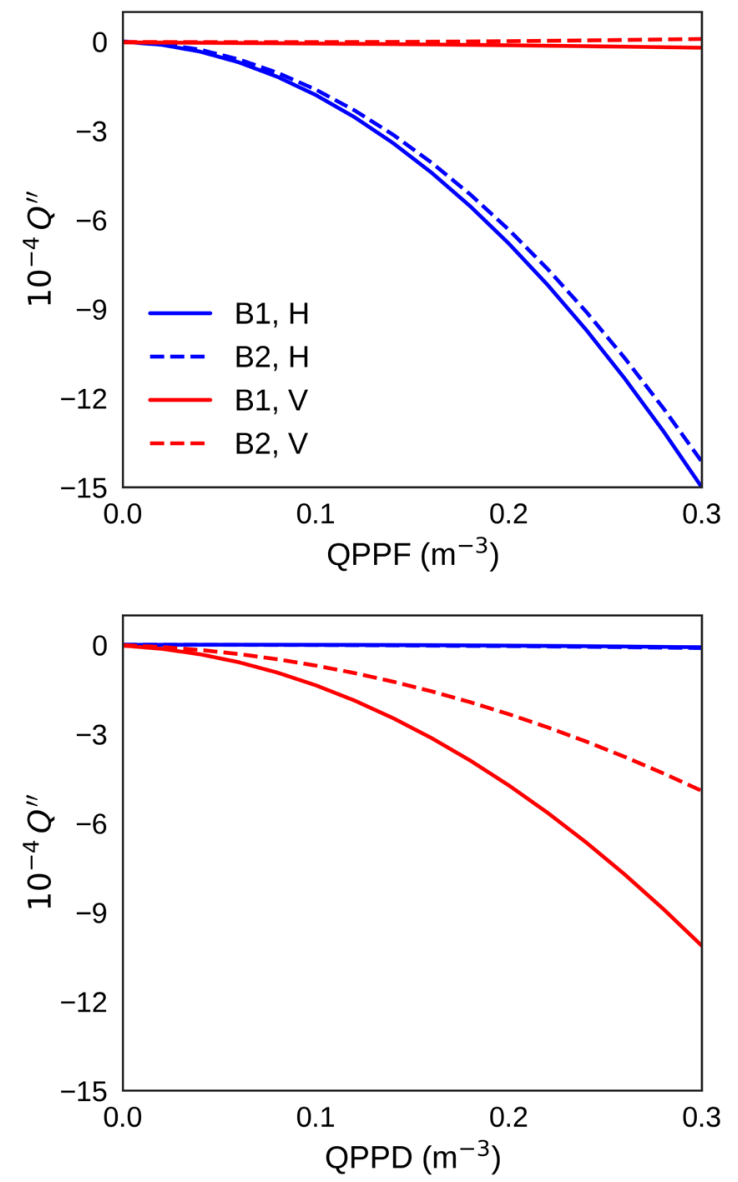

FIG. 2. $Q^{\prime \prime}$ predicted by MAD-X for the two beams ( $B 1$ and $B 2$ ) for the horizontal $(H)$ and the vertical $(V)$ planes as a function of the QPPF (top) and QPPD (bottom) sextupole knobs, respectively. 


\section{B. Detuning with transverse amplitude}

Powering the main sextupoles with the scheme described in the previous section not only introduces $Q^{\prime \prime}$ but also creates non-negligible transverse detuning coefficients $\alpha_{m n}=\partial Q_{m} / \partial\left(2 J_{n}\right)$, with $m, n \in\{x, y\}$ [20]. They give rise to detuning with transverse amplitude and hence Landau damping in the same manner as from magnetic octupoles [4]:

$$
\begin{aligned}
& \Delta Q_{x}\left(J_{x}, J_{y}\right)=\alpha_{x x} 2 J_{x}+\alpha_{x y} 2 J_{y}, \\
& \Delta Q_{y}\left(J_{x}, J_{y}\right)=\alpha_{y y} 2 J_{y}+\alpha_{y x} 2 J_{x},
\end{aligned}
$$

where the cross-detuning coefficients are identical, i.e., $\alpha_{x y}=\alpha_{y x}$. For the studies presented here, the additional detuning with the transverse amplitude is an undesired side effect. After all, the goal is to show that the beam stabilization happens as a result predominantly of detuning with the longitudinal amplitude. The additional transverse detuning coefficients complicate the process of determining the actual origin of the Landau damping, i.e., whether it is mostly a result of detuning with the transverse or longitudinal amplitude. However, as long as the amount of transverse amplitude detuning from the sextupoles is small enough, the two effects can be clearly disentangled by means of tracking simulations (see Sec. V B).

Figure 3 illustrates the amount of detuning with the transverse amplitude introduced by the two sextupole knobs QPPF (left) and QPPD (middle), respectively. The results have been obtained with MAD-X and PTC calculations. Clearly, QPPF introduces mainly $\alpha_{x x}$ and $\alpha_{x y}$, while QPPD gives rise mostly to $\alpha_{y y}$. The results are shown only for beam 1, but they are similar for beam 2. For comparison, also the detuning coefficients introduced by the LHC Landau octupoles are shown in the figure (right). The latter are subdivided into a focusing and a defocusing family, depending on whether they are installed near the focusing or defocusing quadrupoles, respectively. During machine operation, both families are powered with the same absolute current but with opposite signs. For the plot presented here, positive focusing and negative defocusing currents are used. The symbol $I_{\text {oct }}$ corresponds to the current in the focusing family throughout the paper.

\section{C. $Q^{\prime \prime}$ measurement method}

To measure the amount of $Q^{\prime \prime}$ present in the LHC, a sinusoidal frequency modulation is applied to the main $\mathrm{rf}$ system of the machine. This results in a variation of the momentum deviation $d p / p$ of the beam, as illustrated by the top plot in Fig. 4. Because of chromaticity, such a modulation translates into time-varying betatron tunes $Q_{x, y}(t)$ which can be determined from the base-band tune meter measurement (BBQ) [21]. The middle plot in Fig. 4 shows an example of horizontal BBQ spectral data. The fractional tune values $q_{x}$ are obtained from the BBQ turn-by-turn measurement with a sliding window frequency analysis using SUSSIX [22]. Several synchrotron sidebands as well as some noise lines can be seen (yellow). To eliminate systematic errors in the measurement of $Q^{\prime \prime}$, one synchrotron sideband must be selected consistently throughout the modulation period as illustrated by the overlaid red markers. To extract the first and second order chromaticities, the selected tune values are then binned with respect to $d p / p$, and a weighted second order polynomial fit of $Q$ vs $d p / p$ is applied. The result is shown in the bottom plot in Fig. 4. The error bars correspond to the standard deviation of the mean calculated during the binning process.

The main reason for choosing this method is that it provides a fast measurement of the nonlinear chromaticity. Modulation windows of about $60 \mathrm{~s}$ contain enough data to obtain a satisfying result. While usually optics measurements at the LHC are performed with a bunch of only $10^{10} \mathrm{p}$, here the measurement had to be done with bunches of 10 times larger intensities because of time constraints. As a result of the larger bunch charge, the reach in $d p / p$ is limited to about $\pm 3 \times 10^{-4}$ (restricted dynamic aperture), implying a reduced accuracy of the measurement. At these values of $d p / p$, the sensitivity of the betatron tunes to the

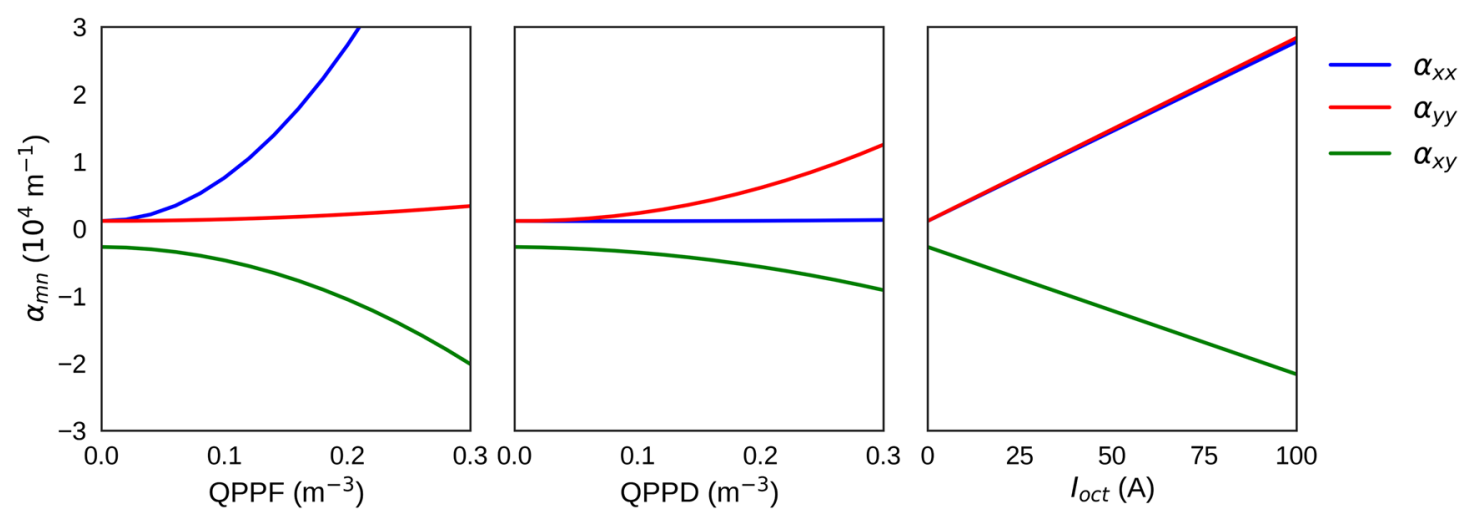

FIG. 3. MAD-X calculation of the transverse amplitude detuning coefficients introduced by the two sextupole knobs QPPF (left) and QPPD (middle) and, for comparison, by the LHC Landau octupoles (right). 

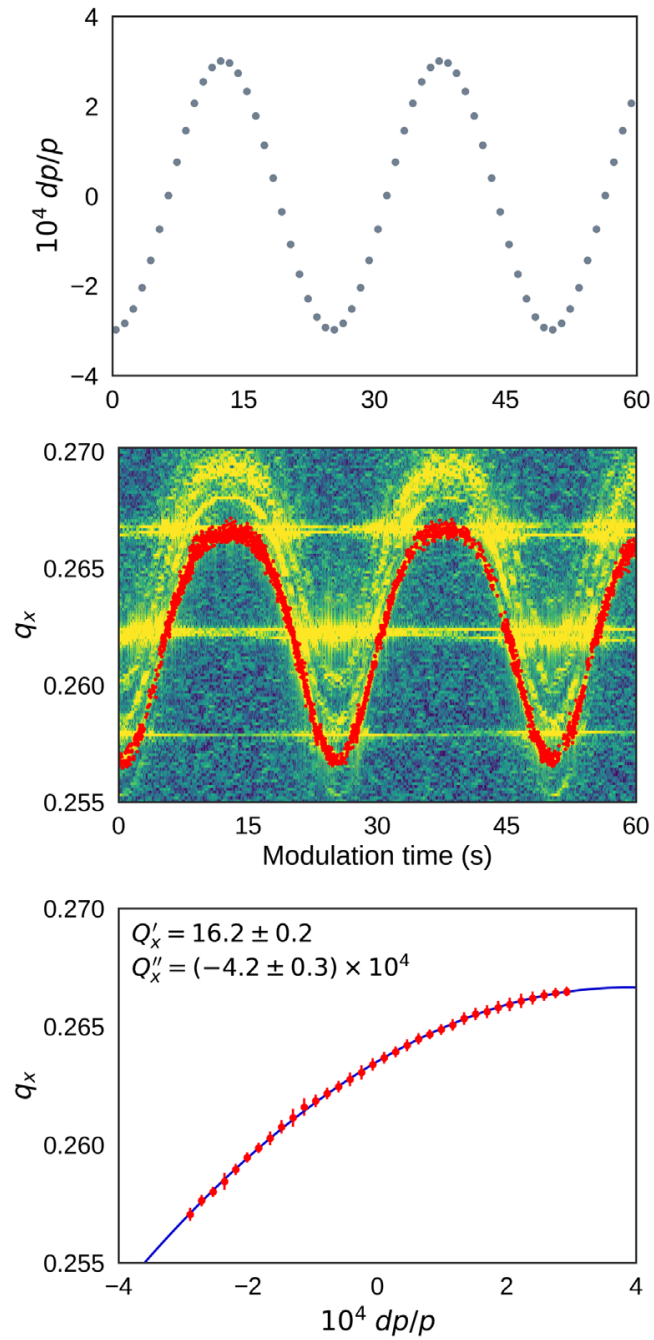

FIG. 4. Top: Variation of the momentum deviation of the beam as a result of the rf frequency modulation. Middle: Horizontal base band tune meter (BBQ) spectral data acquired during momentum modulation, overlaid with the selected SUSSIX tune peaks (red markers). Bottom: Weighted second order polynomial fit (blue line) to the data (red).

higher orders of chromaticity is relatively low. Hence, in particular, for values of $Q^{\prime \prime}$ close to zero, the relative error on the second order chromaticity measurement becomes significant. Furthermore, the widths of the peaks in the frequency spectra, which are particularly large in the presence of the tune spread created by a strong powering of the Landau octupoles, contribute to the uncertainty of the fits as do the noise lines. The Landau octupoles were required during some of the measurements to guarantee beam stability and to determine their contribution to $Q^{\prime \prime}$. The noise is a result mostly of the high bunch intensity used during the measurement.

\section{LHC SINGLE-BUNCH STABILITY AT TOP ENERGY}

This section introduces the single-bunch instability in the LHC observed at $6.5 \mathrm{TeV}$. The most unstable head-tail mode is characterized and its mitigation with the Landau octupoles is explained using both experimental data and PyHEADTAIL simulations.

\section{A. Instability characteristics}

In 2016, a campaign was launched in the LHC to measure the single-bunch stability thresholds at different chromaticities $[6,23]$. At an energy of $6.5 \mathrm{TeV}$, with design bunch parameters, $Q_{x, y}^{\prime}$ between 11 and 14 units, and in the presence of the transverse feedback system with a damping time of $\tau_{\mathrm{fb}} \approx 100$ turns, the most prominent transverse instability was found to be a horizontal head-tail mode with azimuthal and radial numbers $l=0$ and $m=2$, respectively. The upper plot in Fig. 5 shows the corresponding head-tail pattern acquired with the LHC head-tail monitor during the measurement [24]. Using a detailed impedance model of the LHC and a simplified model of the transverse feedback system, macroparticle tracking simulations with PyHEADTAIL predict the same instability as observed in the machine with the correct azimuthal and radial mode numbers. The corresponding head-tail mode pattern is displayed in the lower plot in Fig. 5 and is in good agreement with the pattern observed during the measurement. The main beam, machine, and simulation parameters used for the study are summarized in Table II.

\section{B. Instability mitigation with Landau octupoles}

During routine machine operation, this instability is mitigated by means of the Landau octupoles. During the measurement campaign, the minimum Landau octupole current required to suppress the instability was determined to $I_{\text {oct }}=96_{-10}^{+29}$ A for the beam and machine parameters
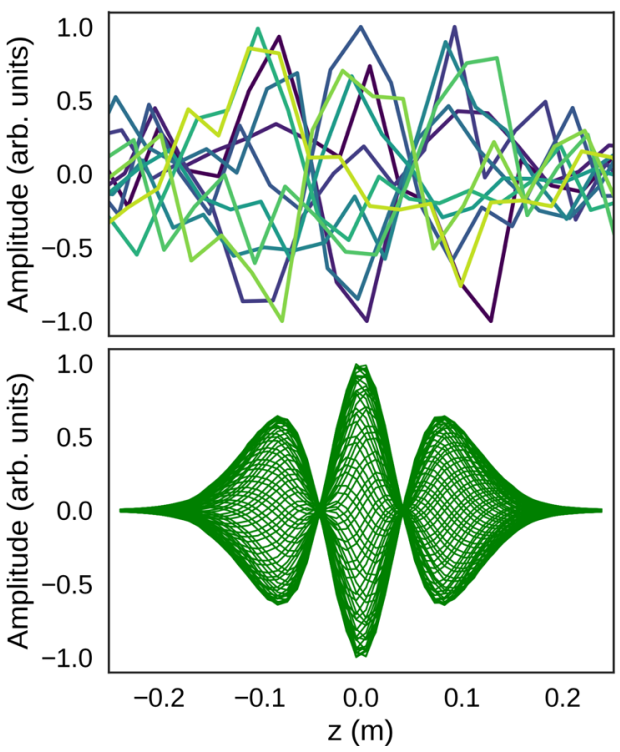

FIG. 5. Head-tail mode patterns from LHC measurements (top) and simulations (bottom) for a single bunch at the top energy $(6.5 \mathrm{TeV})$. 
TABLE II. Main parameters used in PyHEADTAIL to reproduce the LHC experimental machine setup at $6.5 \mathrm{TeV}$.

\begin{tabular}{lcl}
\hline \hline Parameter & Symbol & \multicolumn{1}{c}{ Value } \\
\hline Beam energy & $E$ & $6.5 \mathrm{TeV}$ \\
Bunch intensity & $N_{b}$ & $1.2 \times 10^{11} \mathrm{p}$ \\
Transverse normalized emittance & $\epsilon_{x, y}$ & $2.0 \mu \mathrm{m} \mathrm{rad}$ \\
Bunch length & $4 \sigma_{t}$ & $1.20 \mathrm{~ns}$ \\
Chromaticity & $Q_{x, y}^{\prime}$ & 13 \\
Transverse feedback system & $\tau_{\mathrm{fb}}$ & 100 turns \\
Number of macroparticles & $N_{\mathrm{mp}}$ & $10^{6}$ \\
Number of turns & $N_{t}$ & $5 \times 10^{5}$ turns \\
\hline \hline
\end{tabular}

listed in Table II [6]. The upper error corresponds to the step size used for the scan in the Landau octupole current which was performed from high to low. The lower error originates from the uncertainty in the transverse emittance measurement.

The Landau damping from the octupole magnets in the LHC is mainly due to the incoherent tune spread from detuning with the transverse amplitude. However, there is also an indirect contribution of detuning with the longitudinal amplitude from these magnets. As explained in Sec. III A, $Q^{\prime \prime}$ has a contribution among others from the integral of $D_{x}^{2} k_{3}$ around the accelerator ring. By consequence, the LHC Landau octupoles located in dispersive regions will introduce second order chromaticity with an amount that depends linearly on the octupolar strength $k_{3}$. For this reason, the $Q^{\prime \prime}$ contribution from the Landau octupoles was both measured and simulated for the given optics configuration. For the measurement, the change of $Q^{\prime \prime}$ was determined when increasing the Landau octupole current from $I_{\text {oct }}=40$ A to $I_{\text {oct }}=320 \mathrm{~A}$. The measured amounts of $Q^{\prime \prime}$ introduced per current are summarized in Table III alongside MAD-X predictions. The values obtained from the model are consistent with the measurements, although the latter have rather large uncertainties due to the limited sensitivity of the measurement method (see Sec. III C). It is also worth noting that, in the absence of the Landau octupoles, the $Q_{x, y}^{\prime \prime}$ in the LHC is practically zero, both in measurements and in MAD-X calculations. A comparison is shown in Table IV.

To compare the measured stabilizing Landau octupole current with model predictions, a scan in $I_{\text {oct }}$ has been performed in PyHEADTAIL, again using the parameters in

TABLE III. MAD-X calculations vs measurements of second order chromaticity introduced by the Landau octupoles per current for the two LHC beams $(B)$.

\begin{tabular}{lccccr}
\hline \hline & \multicolumn{2}{c}{$\Delta Q_{x}^{\prime \prime} / \Delta I_{\text {oct }}(1 / \mathrm{A})$} & & \multicolumn{2}{c}{$\Delta Q_{y}^{\prime \prime} / \Delta I_{\text {oct }}(1 / \mathrm{A})$} \\
\cline { 2 - 3 } \cline { 5 - 6 }$B$ & Simulated & Measured & & Simulated & Measured \\
\hline 1 & 49 & $57 \pm 20$ & & -23 & $2 \pm 27$ \\
2 & 48 & $43 \pm 22$ & & -21 & $-7 \pm 28$ \\
\hline \hline
\end{tabular}

TABLE IV. MAD-X calculations vs measurements of second order chromaticity at $6.5 \mathrm{TeV}$ for the two LHC beams $(B)$ and at zero Landau octupole current for operational machine settings.

\begin{tabular}{lccccc}
\hline \hline & \multicolumn{2}{c}{$10^{-4} Q_{x}^{\prime \prime}$} & & \multicolumn{2}{c}{$10^{-4} Q_{y}^{\prime \prime}$} \\
\cline { 2 - 3 } \cline { 5 - 6 }$B$ & Simulated & Measured & & Simulated & Measured \\
\hline 1 & 0.0 & $0.0 \pm 0.2$ & & 0.0 & $0.0 \pm 0.2$ \\
2 & 0.0 & $0.0 \pm 0.2$ & & 0.0 & $0.0 \pm 0.2$ \\
\hline \hline
\end{tabular}

Table II. The contribution from the Landau octupoles to the $Q^{\prime \prime}$ has also been included according to the values quoted in Table III. The final results are summarized in Fig. 6. The plot shows the instability growth rates in the horizontal and the vertical planes as a function of the Landau octupole current. They have been extracted from an exponential fit to the bunch centroid motion. Clearly, with an increasing current, i.e., more tune spread and hence more Landau damping, the growth rates are reduced until the instability is completely suppressed. The gray area marks the region where the beam is stable over the entire simulation period of $N_{t}=5 \times 10^{5}$ turns. This is further illustrated by Fig. 7, where the bunch centroid motion is shown over the full simulation period for four different currents in the Landau octupoles. The stabilizing current lies at $I_{\text {oct }}=$ $107.5 \pm 2.5 \mathrm{~A}$. At this value, the Landau octupoles contribute to second order chromaticity with $\Delta Q_{x}^{\prime \prime} \approx 5000$ and $\Delta Q_{y}^{\prime \prime} \approx-2400$, respectively.

The model predicts a threshold current that is consistent with the measurement in the machine, which demonstrates that the main beam dynamics are well modeled in PyHEADTAIL. In particular, the reliability of the LHC impedance model for single-bunch instabilities is confirmed for the operational machine configuration at

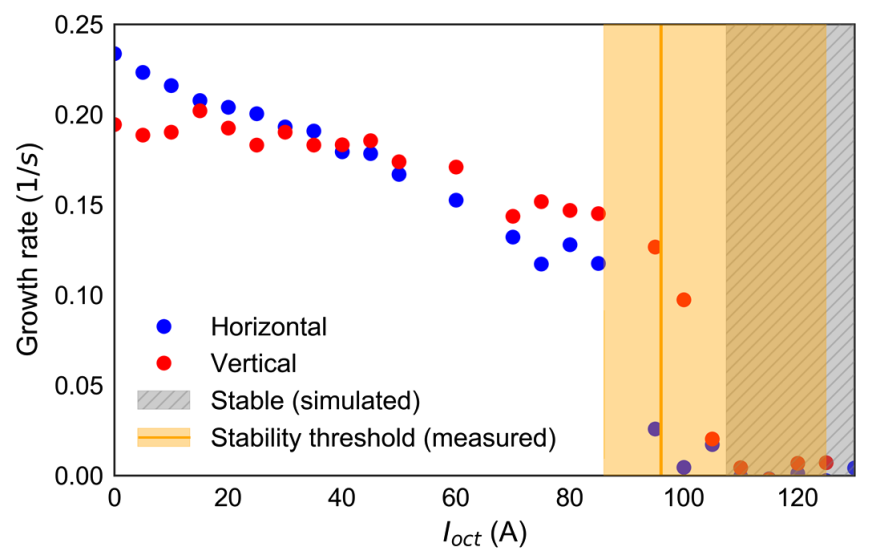

FIG. 6. PyHEADTAIL simulations showing the stabilization of a single bunch with Landau octupoles in the LHC at $6.5 \mathrm{TeV}$. Centroid growth rates vs Landau octupole current are shown, with the gray area indicating the cases that were stable over $N_{t}=5 \times 10^{5}$ turns (compare Fig. 7). 


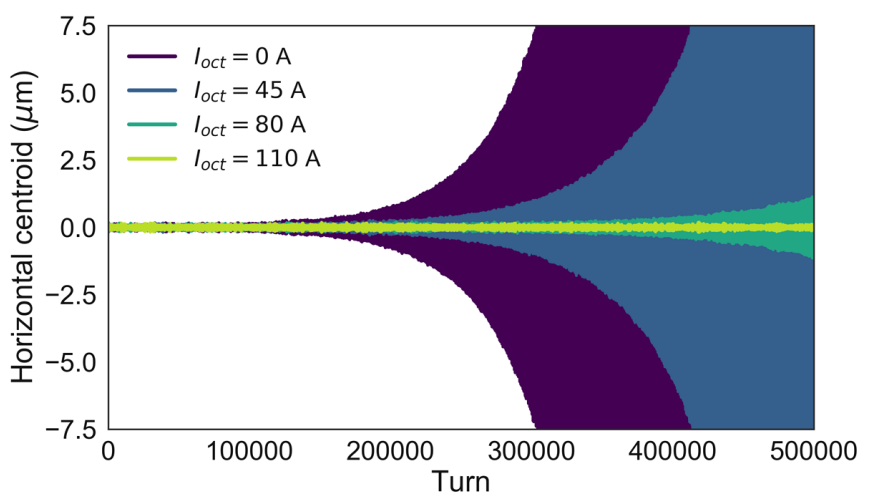

FIG. 7. Horizontal bunch centroid signals over the full PyHEADTAIL simulation period for a selection of four different Landau octupole currents for the single-bunch head-tail instability observed in the $\mathrm{LHC}$ at $6.5 \mathrm{TeV}$.

6.5 TeV, which will also be used in the following for the stability studies with second order chromaticity.

\section{LHC SINGLE-BUNCH STABILITY STUDIES WITH $Q^{\prime \prime}$}

This section discusses the experiments and simulations carried out with $Q^{\prime \prime}$ in the LHC. The experimental procedure and the observations made during the machine development session are described and explained using PyHEADTAIL simulations. The aim of the section is to demonstrate stabilization of single bunches through Landau damping predominantly from detuning with the longitudinal rather than the transverse amplitude in both experiments and simulations.

\section{A. Experimental procedure and observations}

The experiment was performed with two bunches in each of the two LHC beams at an energy of 6.5 TeV. The main parameters of the four individual bunches, i.e., the bucket number $(\mathrm{Bkt})$, bunch intensity $\left(N_{b}\right)$, four-sigma bunch length $\left(4 \sigma_{t}\right)$, and the transverse normalized emittances $\left(\epsilon_{x, y}\right)$, are listed in Table $\mathrm{V}$. Because of the presence of the transverse bunch-by-bunch feedback system as well as the large longitudinal separation of the two bunches in each beam, coupled-bunch wakefield effects can be neglected. At the beginning of the experiment, the Landau octupoles

TABLE V. Initial bunch parameters of the four individual bunches in the two beams $(B)$ residing in different buckets (Bkt) while performing the experiments with $Q^{\prime \prime}$.

\begin{tabular}{rrcccc}
\hline \hline$B$ & $\mathrm{Bkt}$ & $N_{b}\left(10^{11} \mathrm{p}\right)$ & $4 \sigma_{t}(\mathrm{~ns})$ & $\epsilon_{x}(\mu \mathrm{m} \mathrm{rad})$ & $\epsilon_{y}(\mu \mathrm{m} \mathrm{rad})$ \\
\hline 1 & 0 & $0.91 \pm 0.01$ & $1.04 \pm 0.02$ & $1.5 \pm 0.2$ & $1.8 \pm 0.2$ \\
& 700 & $1.08 \pm 0.01$ & $1.06 \pm 0.02$ & $1.9 \pm 0.1$ & $1.7 \pm 0.1$ \\
2 & 30 & $0.92 \pm 0.01$ & $1.04 \pm 0.02$ & $1.2 \pm 0.1$ & $1.5 \pm 0.1$ \\
& 740 & $1.14 \pm 0.01$ & $1.06 \pm 0.02$ & $1.2 \pm 0.1$ & $1.3 \pm 0.1$ \\
\hline \hline
\end{tabular}

were powered with a current of $I_{\text {oct }}=320$ A to guarantee beam stability. To test stabilization with $Q^{\prime \prime}$, the aim was to set QPPF and QPPD such that $Q_{x, y}^{\prime \prime} \approx-4 \times 10^{4}$ in both beams once the current in the Landau octupoles would be reduced to zero. Because of the strong current, the additional contribution to the second order chromaticity from the Landau octupoles in dispersive regions was significant and, hence, had to be taken into account during the $Q^{\prime \prime}$ adjustment process. At the initial current of $I_{\text {oct }}=320 \mathrm{~A}$, for example, they were $\Delta Q_{x}^{\prime \prime} \approx 1.5 \times 10^{4}$ and $\Delta Q_{y}^{\prime \prime} \approx-0.7 \times$ $10^{4}$ according to Table III. To introduce the desired amount of $Q^{\prime \prime}$, several iterations were made by varying the sextupole knobs QPPF and QPPD, remeasuring $Q^{\prime \prime}$, and comparing the results to MAD-X calculations.

Once the targeted sextupole settings were reached, the current in the Landau octupoles was reduced in steps of 40 A from 320 down to 0 A. At $I_{\text {oct }}=40 \mathrm{~A}$, all four bunches were still stable. This already indicated a successful stabilizing effect from $Q_{x, y}^{\prime \prime}$, recalling that $I_{\text {oct }}=96_{-10}^{+29} \mathrm{~A}$ was necessary to control the instability without second order chromaticity. At this point, a chromaticity measurement was performed, since the effect from the Landau octupoles was small enough to allow for an accurate assessment of $Q^{\prime \prime}$. The values are summarized in Table VI and compared to MAD-X calculations after the subtraction of the remaining contribution from the Landau octupoles $\left(\Delta Q_{x}^{\prime \prime} \approx 1.9 \times 10^{3}\right.$ and $\left.\Delta Q_{y}^{\prime \prime} \approx-0.9 \times 10^{3}\right)$. They demonstrate good agreement and a successful adjustment procedure for $Q^{\prime \prime}$ in the machine. The amount of second order chromaticity was consistent with the targeted $-4 \times 10^{4}$ units in three out of four planes, while it was, in absolute terms, slightly lower than expected in the horizontal plane of beam 2 .

When the Landau octupole current was reduced further, from 40 to $0 \mathrm{~A}$, a horizontal instability occurred in beam 1 for the bunch residing in bucket 700, while the other three bunches remained stable. The reasons will be discussed in Sec. V B. For the unstable bunch, the observed head-tail instability was an azimuthal mode $l=-1$. The top right plot in Fig. 8 displays the turn-by-turn traces acquired with the LHC head-tail monitor for the unstable bunch. It shows a pattern with three nodes, indicating a radial mode $m=3$. The characteristics of this instability are clearly different

TABLE VI. $Q^{\prime \prime}$ MAD-X calculations vs measurements for the two LHC beams $(B)$ with powering of the main sextupoles (MS) at zero Landau octupole current after the $Q^{\prime \prime}$ adjustment procedure targeting an amount of $Q_{x, y}^{\prime \prime}=-4 \times 10^{4}$ units in both beams.

\begin{tabular}{|c|c|c|c|c|c|c|}
\hline \multirow[b]{2}{*}{$B$} & \multicolumn{2}{|c|}{$\operatorname{MS}\left(\mathrm{m}^{-3}\right)$} & \multicolumn{2}{|c|}{$10^{-4} Q_{x}^{\prime \prime}$} & \multicolumn{2}{|c|}{$10^{-4} Q_{y}^{\prime \prime}$} \\
\hline & QPPF & QPPD & Simulated & Measured & Simulated & Measured \\
\hline 1 & 0.15 & 0.19 & -3.8 & $-4.4 \pm 0.6$ & -3.9 & $-4.0 \pm 0.6$ \\
\hline 2 & 0.15 & 0.26 & -3.7 & $-3.1 \pm 0.5$ & -3.7 & $-3.7 \pm 0.6$ \\
\hline
\end{tabular}



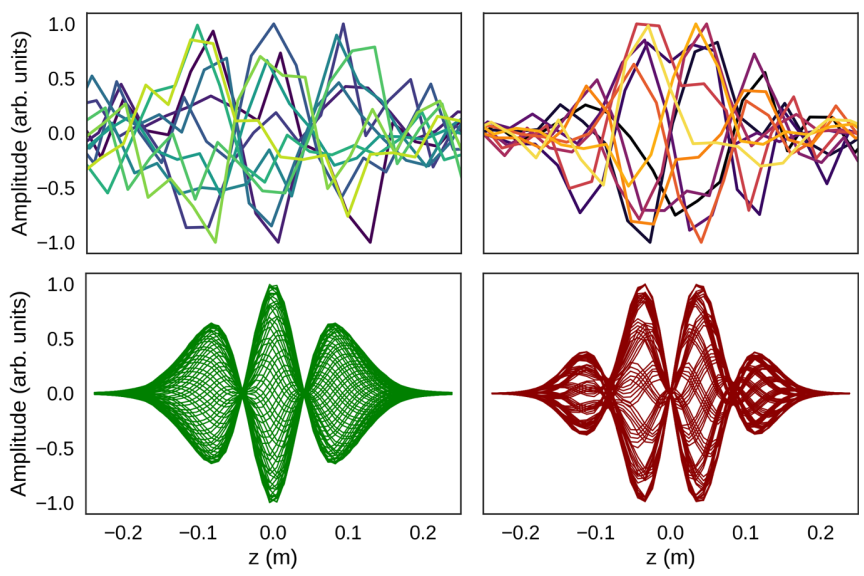

FIG. 8. Comparison of the two modes observed in the machine (top) and in PyHEADTAIL simulations (bottom) without (left) and with (right) the effects from $Q^{\prime \prime}$. They correspond to the working points labeled (a) and (b), respectively (compare Fig. 9).

from those described in Sec. IVA, where an azimuthal mode $l=0$ with two nodes in the head-tail pattern was recorded (Fig. 8, top left). The main difference with respect to the situation in the previous section is the large amount of $Q_{x, y}^{\prime \prime}$ that was now present in the machine. The reasons for the change of the head-tail mode are explained with PyHEADTAIL simulations in the following Sec. V B.

\section{B. PyHEAdTAIL simulations and interpretation}

There are three main goals for the simulation studies: (i) whether the stabilization of three out of four bunches is indeed a result of mostly detuning with the longitudinal amplitude, or whether it could have been provided by the transverse detuning coefficients $\alpha_{m n}$, with $m, n \in\{x, y\}$, introduced parasitically by the QPPF and QPPD knobs, (ii) to understand why one of the four bunches became unstable, and (iii) why the observed head-tail mode with nonzero $Q^{\prime \prime}$ knobs is now an azimuthal mode $l=-1$ rather than an $l=0$ as originally observed in Sec. IVA.

At the experimental working point $\mathrm{QPPF}=0.15 \mathrm{~m}^{-3}$ and $\mathrm{QPPD}=0.19 \mathrm{~m}^{-3}$ (beam 1), the transverse detuning coefficients generated by the sextupoles are comparable to LHC Landau octupole currents of $I_{\text {oct }} \approx 50 \mathrm{~A}\left(\alpha_{x x}\right)$, $I_{\mathrm{oct}} \approx 20 \mathrm{~A},\left(\alpha_{y y}\right)$ and $I_{\mathrm{oct}} \approx 30 \mathrm{~A}\left(\alpha_{x y}\right)$, respectively (compare Fig. 3). They are all well below the above-mentioned single-bunch stability threshold of $I_{\text {oct }}=96_{-10}^{+29} \mathrm{~A}$, which indicates that an important contribution to beam stability must have been provided by $Q^{\prime \prime}$. To understand the horizontal instability observed in one of the bunches, however, simulation studies were required. Optics results from MAD-X, among them the dependencies of $Q_{x, y}^{\prime \prime}$ and the transverse detuning coefficients on the QPPF and QPPD knobs, displayed in Figs. 2 and 3, are also included in PyHEADTAIL such that both the stabilizing effects from detuning with the transverse and longitudinal amplitudes are modeled. In all the studies, the Landau octupoles are switched off to reproduce the situation of the experiment at $I_{\text {oct }}=0$ A where the bunch became unstable. The bunch parameters are taken from the measurements in Table $\mathrm{V}$ for the bunch in beam 1 , bucket $700 . N_{\mathrm{mp}}=4 \times 10^{5}$ macroparticles are tracked over $N_{t}=1.8 \times 10^{6}$ turns using again the same impedance model of the LHC.

In a first set of simulations, the tune spread from $Q^{\prime \prime}$ is deliberately excluded. This is to assess whether the spread from the transverse detuning coefficients alone, which is introduced parasitically by the QPPF and QPPD knobs, would be enough to provide stability at the working point $\left(\mathrm{QPPF}=0.15 \mathrm{~m}^{-3}, \mathrm{QPPD}=0.19 \mathrm{~m}^{-3}\right)$. The results are summarized in the upper plots in Fig. 9 for the horizontal (left) and the vertical (right) planes. The color code shows the relative emittance growth over the simulation period (in percent), where blue means stable and white means unstable. The dots represent the azimuthal mode number of the instability predicted for each setting of (QPPF, QPPD) in case the bunch was unstable in the simulation. The two working points labeled (a) and (b) show the situation with and without the $Q^{\prime \prime}$ knobs and correspond to the experimental machine configuration described in Secs. IVA and VA, respectively. For the latter, the error bars correspond to the uncertainty in the $Q_{x, y}^{\prime \prime}$ listed in Table VI. Most of the area is unstable, in particular, the working point (b) where the $Q^{\prime \prime}$ measurements of Sec. VA were carried out. This demonstrates that the transverse detuning coefficients from the sextupole knobs indeed do not provide sufficient Landau damping. The experiment showed that three out of four bunches remained stable, while the model predicts instability with a large level of significance. Furthermore, the instability predicted by the model is an azimuthal mode $l=0$ (green dots) with two nodes in the head-tail pattern, which is not consistent with the experimental observations made in the machine at the working point (b). Rather, an azimuthal mode $l=-1$ with three nodes is what was observed experimentally (Fig. 8, right).

The second set of simulations is shown in the two lower plots in Fig. 9. This simulation now also includes the effects from detuning with the longitudinal amplitude as introduced by the second order chromaticity. Two main observations can be made. First, large regions of stability (blue) are created in the QPPF vs QPPD plane. The two main stable areas are separated by an unstable band in both the horizontal and the vertical planes which shows a different head-tail mode with an azimuthal number $l=-1$ (red dots). The reason for that is that second order chromaticity changes the effective impedance, similarly to a first order chromaticity. This effect is described by the Vlasov formalism [2] and is currently under more detailed analytical study. It affects the complex coherent tune shift and can also change the most unstable mode. The bottom plots in Fig. 9 are hence a combination of a change in effective impedance and Landau damping, both introduced by $Q^{\prime \prime}$. 



FIG. 9. Two PyHEADTAIL studies showing the predicted headtail instabilities as a function of the sextupole knobs QPPF and QPPD. Labels (a) and (b) mark the two working points used during the experiments. Top: Results obtained without $Q_{x, y}^{\prime \prime}$ effects but including the transverse amplitude detuning coefficients from the sextupole knobs. Bottom: Results obtained including also $Q_{x, y}^{\prime \prime}$ effects.

The first unstable band observed at low values of QPPF (QPPD) is the azimuthal mode zero (green dots), consistent with the experimental measurements described in Sec. IVA. The stable region between the two unstable bands $l=0$ and $l=-1$ arises from sufficient Landau damping of both modes. The further increase of QPPF (or QPPD), however, leads to a change of the effective impedance, in a way that Landau damping is lost for the $l=-1$ mode. For even larger amounts of $Q^{\prime \prime}$, however, all the instabilities can be suppressed. The second observation is that the working point (b), although essentially stable, lies very close to the unstable band of the $l=-1$ mode. Indeed, the experimental data clearly revealed the observed horizontal instability to be of mode $l=-1$. The LHC headtail monitor signal is in good agreement with the predictions from simulations. Both of them feature a three-node coherent oscillation pattern along the bunch as shown in Fig. 8 on the right. Thus, the experiment and simulation agree both in the azimuthal as well as the radial mode numbers of the excited instability.

The simulation model cannot make a definite statement about why only one of the four bunches became unstable during the experiment. This is mostly due to the measurement uncertainties in the bunch length, intensity, and emittance and to some extent also due to the limited accuracy of the impedance model and, therefore, the uncertainty on the beam stability predictions from PyHEADTAIL. A number of considerations can be made, however, to better understand and explain the experimental observations. First, the bunches in beam 1 and beam 2 do not experience the same amount of Landau damping due to different settings in $Q_{x, y}^{\prime \prime}$ for the two beams (Table VI). While this may explain why beam 1 and beam 2 behave differently, it does not answer the question why the two bunches in beam 1 exhibit a different behavior. After all, they are both subject to the same amount of second order chromaticity. The only remaining obvious differences between the two bunches are their transverse emittances and intensities listed in Table V. The unstable bunch residing in bucket 700 had a slightly larger horizontal emittance compared to the stable one located in bucket $0(1.9 \pm 0.1$ vs $1.5 \pm 0.2 \mu \mathrm{m} \mathrm{rad})$. The vertical emittances, on the other hand, were identical within the errors ( $1.7 \pm 0.1 \mathrm{vs} 1.8 \pm 0.2 \mu \mathrm{m} \mathrm{rad})$. As a result of the larger horizontal emittance, the Landau damping introduced by the parasitic transverse detuning coefficient $\alpha_{x x}$ in the horizontal plane is actually larger for the unstable bunch compared to the stable one. However, the difference between the emittances is minor, and, more importantly, the transverse detuning coefficient is very small. It is clear from the upper plots in Fig. 9 that the Landau damping from detuning with the transverse amplitude is insignificant for the instability under consideration. The bunch intensities, on the other hand, are the most likely explanation for the differences observed in terms of bunch stability. The stable bunch had a lower intensity of $(0.91 \pm 0.01) \times 10^{11} \mathrm{p}$ compared to $(1.08 \pm 0.01) \times 10^{11} \mathrm{p}$ for the unstable one. An impedance-driven instability is more difficult to be stabilized for a bunch of higher intensity, since its complex coherent tune shift is larger and more likely to lie outside the stability diagram.

In summary, the experiments can be reproduced in simulations only when including all the effects of second order chromaticity in PyHEADTAIL. This concerns both the Landau damping effect as well as the change of the effective impedance, which manifests as a change of the azimuthal and radial mode numbers. When including the effects of the second order chromaticity in the model, the observed head-tail modes are consistent with simulations at both working points (a) and (b). Moreover, the second order chromaticity is the main stabilizing effect, while the contribution from detuning with the transverse amplitude introduced by the sextupole knobs plays a minor role here. The reason why one of the four bunches has gone unstable during the experiment is explained by the differences in bunch intensities as well as in the second order chromaticities present in the two beams.

\section{CONCLUSIONS AND OUTLOOK}

Detuning with the longitudinal amplitude is potentially a usable technique to introduce Landau damping in the 
transverse planes, particularly for the high-brightness, low transverse emittance beams of future hadron colliders where conventional approaches, such as magnetic octupoles, may not provide a sufficient tune spread. Second order chromaticity or an $\mathrm{rf}$ quadrupole cavity are two possible ways to introduce a betatron tune spread as a function of the longitudinal action, and it was shown analytically that the two mechanisms are equivalent in a first approximation. The second order chromaticity has the advantage that it can be introduced in a machine like the LHC by using the existing hardware and changing the optics of the accelerator. In that way, it can be used to experimentally study the stabilization from transverse detuning with the longitudinal amplitude in a cost-effective manner. The LHC represents an ideal accelerator environment for such a proof-of-principle experiment, as it is a well-studied machine, in terms of both optics and collective effects. Here, a sextupole powering scheme was employed to control $Q^{\prime \prime}$ in both beams and the two transverse planes independently. Beam measurements and MAD-X calculations show good agreement and demonstrate a thorough control of the second order chromaticity in the LHC.

The single-bunch stability in the LHC at $6.5 \mathrm{TeV}$ has been recapped, and the stabilizing Landau octupole currents for the most unstable head-tail mode were shown to be consistent between experiments and PyHEADTAIL simulations. Based on the same machine configuration, experiments have been performed to assess the stabilization of single bunches with second order chromaticity. Beam dynamics simulations clarify that detuning from the transverse amplitude alone, introduced parasitically by the sextupole knobs, cannot explain the observations made in the machine. Clearly, $Q^{\prime \prime}$ makes a strong contribution to the beam stability as demonstrated by the combined analysis of the data and PyHEADTAIL simulations. Furthermore, the second order chromaticity leads to a change of the head-tail mode, which is expected from the Vlasov theory and consistently observed in simulations and experiments conducted here. This is due to a change of the effective impedance introduced by chromaticity, and a detailed analysis will be reported in a forthcoming paper.

With the present LHC beam parameters, the magnetic octupoles provide enough Landau damping such that no additional means are required. Nevertheless, the work on beam stabilization from detuning with the longitudinal amplitude will be continued. Future studies will, among others, assess the effect of $Q^{\prime \prime}$ on the dynamic aperture (beam lifetime), its potential for stabilization of, e.g., electron cloud-driven instabilities, and the evaluation of various $Q^{\prime \prime}$ production schemes. A step in that direction has been made recently with a machine development session in the LHC to address the aforementioned questions [25]. However, the scheme that was employed created a large off-momentum beta beating around the ring and hence was not machine-safe for multibunch operation. The offline analysis showed, however, that this may be overcome by a change of the off-momentum collimation scheme. On the other hand, it may also be an indication of why an $\mathrm{rf}$ quadrupole could be a better choice for Landau damping from detuning with the longitudinal amplitude. Other than $Q^{\prime \prime}$, it does not depend on optics constraints but works instead as an independent device. The advantages and disadvantages of both methods, however, need to be evaluated in more detail, especially in terms of singleparticle effects (e.g., dynamic aperture and resonances).

\section{ACKNOWLEDGMENTS}

The authors thank the BE-OP-LHC teams and Machine Development coordinators and participants at CERN for helping with the experiments. Furthermore, they acknowledge Stéphane Fartoukh, Antoine Maillard, and Giovanni Rumolo for fruitful discussions and inputs to these studies.

[1] L. D. Landau, On the vibrations of the electronic plasma, Zh. Eksp. Teor. Fiz. 16, 574 (1946) [J. Phys. USSR 10, 25 (1946)].

[2] A. Chao, Physics of Collective Beam Instabilities in High Energy Accelerators, Wiley Series in Beam Physics and Accelerator Technology (Wiley, New York, 1993).

[3] O. S. Brüning, P. Collier, P. Lebrun, S. Myers, R. Ostojic, J. Poole, and P. Proudlock, LHC Design Report, CERN Yellow Reports: Monographs (CERN, Geneva, 2004).

[4] J. Gareyte, J.-P. Koutchouk, and F. Ruggiero, Technical Report Nos. LHC-Project-Report-91, CERN-LHC-ProjectReport-91, CERN, 1997.

[5] S. Peggs and T. Satogata, Introduction to Accelerator Dynamics (Cambridge University Press, Cambridge, England, 2017), Chap. 10, pp. 107-109.

[6] L. R. Carver et al., in Proceedings of the 7th International Particle Accelerator Conference (IPAC'16), Busan, Korea, 2016 (JACoW, Geneva, 2016), pp. 1434-1437.

[7] E. Métral et al., in Proceedings of the 57th ICFA Advanced Beam Dynamics Workshop on High-Intensity and HighBrightness Hadron Beams (HB'16), Malmö, Sweden, 2016 (JACoW, Geneva, Switzerland, 2016), pp. 254-259.

[8] A. Grudiev, Radio frequency quadrupole for Landau damping in accelerators, Phys. Rev. Accel. Beams 17, 011001 (2014).

[9] K. Papke and A. Grudiev, Design of an rf quadrupole for Landau damping, Phys. Rev. Accel. Beams 20, 082001 (2017).

[10] M. Schenk, A. Grudiev, K. Li, and K. Papke, Analysis of transverse beam stabilization with radio frequency quadrupoles, Phys. Rev. Accel. Beams 20, 104402 (2017).

[11] G. Apollinari, I. Béjar Alonso, O. Brüning, M. Lamont, and L. Rossi, High-Luminosity Large Hadron Collider (HL-LHC): Preliminary Design Report, CERN Yellow Reports: Monographs (CERN, Geneva, 2015).

[12] M. Benedikt and F. Zimmermann, Technical Report No. CERN-ACC-2015-0164, CERN, 2015. 
[13] E. Métral et al., Beam instabilities in hadron synchrotrons, IEEE Trans. Nucl. Sci. 63, 1001 (2016).

[14] Methodical Accelerator Design (MAD), http://mad.web .cern.ch/mad/.

[15] J. S. Berg and F. Ruggiero, in Proceedings of the 1997 Particle Accelerator Conference (Cat. No.97CH36167) (IEEE, New York, 1997), Vol. 2, pp. 1712-1714.

[16] F. C. Iselin, Technical Report No. SL-92-199, CERN, 1994.

[17] S. D. Fartoukh, Technical Report No. CERN-LHC-ProjectReport-308, CERN, 1999.

[18] N. Mounet (unpublished).

[19] F. Schmidt, E. Forest, and E. McIntosh, Technical Report Nos. CERN-SL-2002-044-AP, KEK-REPORT-2002-3, CERN, 2002.

[20] S. Y. Lee, Accelerator Physics, 3rd ed. (World Scientific, Singapore, 2012), Chap. 2, pp. 197-198.

[21] M. Gasior and R. Jones, Technical Report No. LHCProject-Report-853, CERN, 2005.
[22] R. Bartolini and F. Schmidt, Technical Report No. SLNote-98-017-AP, CERN, 1998.

[23] L. R. Carver, D. Amorim, N. Biancacci, X. Buffat, G. Iadarola, K. Łasocha, K. Li, T. Levens, E. Métral, B. Salvant, and C. Tambasco, MD1228: Validation of single bunch stability threshold \& MD1751: Instability studies with a single beam, Technical Report No. CERN-ACCNOTE-2017-0013, CERN, 2017.

[24] T. Levens, K. Łasocha, and T. Lefèvre, in Proc. of International Beam Instrumentation Conference (IBIC'16), Barcelona, Spain, Sept. 13-18, 2016, International Beam Instrumentation Conference No. 5 (JACoW, Geneva, Switzerland, 2017), p. THAL02, .

[25] M. Schenk, S. D. Fartoukh, K. Li, L. Malina, E. Métral, and R. Tomás Garcia, MD2190: Q" Stabilization during injection, Technical Report No. CERN-ACC-NOTE-20180003, CERN, 2018. 